\title{
Aprender historia del arte mediante salidas didácticas. Una experiencia en educación superior
}

\author{
Doi:https://doi.org/10.25100/hye.v15i53.8660 \\ Artículo recibido: 19-02-2018 Artículo aceptado: 28-08-2019
}

\section{Juan Ramón Moreno-Vera}

Profesor ayudante, Doctor. Grupo de investigación DICSO. Dpto. de Didáctica de las Ciencias Matemáticas y Sociales. Facultad de Educación. Universidad de Murcia. Campus de Espinardo, Murcia (España).

Universidad de Murcia, España.

Correo electrónico:jr.moreno@um.es

ORCID: 0000-0002-5395-5981

\section{José Monteagudo Fernández}

Profesor contratado, Doctor. Grupo de investigación DICSO. Dpto. de Didáctica de las Ciencias Matemáticas y Sociales. Facultad de Educación.

Universidad de Murcia. Campus de Espinardo, Murcia, España.

Correo electrónico: jose.monteagudo@um.es

ORCID: 0000-0003-2680-7622

Forma de citar este artículo: Monteagudo Fernández, José y Juan Ramón Moreno-Vera. “Aprender historia del arte mediante salidas didácticas. Una experiencia en educación superior”. Historiay Espacio, vol. 15 nº 53 (2019): 249-270. Doi.org/10.25100/hye.v15i53.8660.

Artículo Tipo 1: de investigación. 
Aprender historia del arte mediante salidas didácticas. Una experiencia en educación superior

Juan Ramón Moreno-Vera - José Monteagudo Fernández

\section{Aprender historia del arte mediante salidas didácticas. Una experiencia en educación superior}

Resumen: En la experiencia que aquí se presenta, estudiantes de la Universidad de Murcia dejaron las aulas por unas horas para llevar a cabo un aprendizaje situado de la historia del arte mediante un itinerario didáctico sobre la Murcia medieval. De este modo, se rompen las barreras físicas del aula y se integran los elementos de aprendizaje con el propio paisaje urbano por donde los estudiantes pasan a diario. El itinerario didáctico partía desde el antiguo Alcázar Seguir de la ciudad, hoy Convento de clausura, pasando por la plaza de Santo Domingo, antigua plaza del zoco; la conjunción urbana de "las cuatro esquinas", donde convergen las calles de los principales gremios de la ciudad; la catedral de Murcia, donde se conservan los restos de la antigua mezquita Al-Jama y, finalmente, en el conjunto monumental de San Juan de Dios, donde aún es visible el nicho del mihrab del antiguo oratorio del Alcázar mayor medieval. Los resultados de la investigación se basan en el análisis de un pre-test y un post-test que el alumnado contestó sobre sus conocimientos de la Murcia medieval. La progresión de aprendizaje del alumnado participante fue positiva mejorando en el post-test los resultados previos.

Palabras clave: Enseñanza de la historia del arte, Edad Media, Patrimonio cultural, Itinerario didáctico.

\section{Learn art history through educational outings. An experience in higher education}

Abstract: In the experience that we present here, students from the University of Murcia left the classrooms for a few hours to carry out a learning located in the history of art through a didactic itinerary on the city of medieval Murcia. In this way, the physical barriers of the classroom are broken and the elements of learning are integrated with the urban landscape through which students pass on to daily. The didactic itinerary started from the city old Kashba, nowadays an enclosed convent, passing through Plaza de Santo Domingo, the old souk plaza; the urban conjunction of "the four corners", where the streets of the main guilds of the city converge; the Cathedral of Murcia, where the remains of the old Al-Jama mosque are preserved; and, finally, in the Monumental Ensemble of San Juan de Dios, where the niche of the mihrab of the old oratory of the medieval Major Kashba is still visible. The results of the research are based on the analysis of a pre-test and a post-test that the students answered about their knowledge of medieval Murcia. The learning progression of the participating students was positive, improving the previous results they had obtained in the pre-test.

Keywords: Art History teaching, Middle Age, Cultural heritage, Didactic itinerary. 


\section{Juan Ramón Moreno-Vera- José Monteagudo Fernández \\ Aprender historia del arte mediante salidas didácticas. Una experiencia en educación superior}

\section{Introducción}

La asignatura de Historia del Arte ha tenido, en el sistema educativo español, una presencia escasa y marginal, pues no existe como tal asignatura en toda la educación obligatoria, siendo un apéndice en las asignaturas de Ciencias Sociales. Incluso en el bachillerato resultante de la última reforma educativa, solamente el alumnado que se incline por la modalidad de Humanidades y Ciencias Sociales tiene la opción de cursarla. De este modo, la mayoría del alumnado, incluido el de letras, termina su paso por el sistema educativo sin haber tenido prácticamente contacto directo con la materia.

Esta realidad contrasta con la propia normativa, en la que se aboga por una educación en historia del arte con la introducción de la competencia en conciencia y expresiones culturales y objetivos que persiguen el conocimiento, el valor y el respeto por los aspectos básicos de la cultura, el patrimonio artístico y cultural e, incluso, la comprensión del lenguaje de las distintas manifestaciones artísticas.

Teniendo presente esta contradicción, la necesidad de una asignatura como Historia del arte viene amparada por los beneficios educativos que puede aportar como herramienta para la formación integral del educando, como alumno, persona y ciudadano. A través de ella, el alumnado tiene la posibilidad de acceder y conocer un nuevo lenguaje, desarrollar su sensibilidad e incluso formar un juicio estético personal. Además, permite la formación de la identidad cultural del alumnado y el conocimiento del patrimonio universal, nacional y local favoreciendo su valoración y conservación.

En opinión de Lozano, la formación en historia del arte y, a través de ella, en el patrimonio, supone reconocer el pasado, aprender a observar los restos materiales paraleerlos, analizarlos e interpretarlos y educar la mirada para entendery comparar lo que se ve ${ }^{1}$, y ello es factible en todos los niveles educativos, incluido el universitario. De ahí que nuestra intención con el alumnado de tercer curso del grado en Educación Primaria en la Universidad de Murcia en la asignatura de Metodología Didáctica para la Enseñanza de las Ciencias Sociales haya sido 
la de proporcionarle unos conocimientos básicos y directos sobre la historia, el arte y el patrimonio de la ciudad de Murcia a través de una salida didáctica del aula.

Las salidas escolares han constituido durante el siglo XX, hasta el decenio de los 80, una característica fundamental de la pedagogía progresista. Muchos e importantes pedagogos de la pasada centuria defendieron la necesidad de establecer lazos de unión entre las aulas y el medio exterior, a través del contacto directo del alumnado con su entorno, para garantizar el éxito de la escolarización obligatoria. Sostuvieron la experiencia como base del aprendizaje, promoviendo el acercamiento de la escuela al mundo real a través de las salidas. Entendían que era la mejor manera de potenciar la observación directa y la vivencia como motivaciones para conseguir un aprendizaje que posibilitase el desarrollo de la personalidad de los alumnos y su integración en la comunidad ${ }^{2}$. La institución de Libre Enseñanza, por ejemplo, utilizaba una metodología para la enseñanza de la naturaleza y del patrimonio históricoartístico que tenía un elemento esencial en las salidas, paseos escolares, excursiones cortas y viajes de larga duración, siendo la visita a museos el núcleo fundamental de las salidas más breves ${ }^{3}$.

Autoras como Vilarrasa ${ }^{4}$ atribuyen a las salidas escolares una gran importancia en la medida en que ayudan a enriquecer la experiencia vivida mediante una toma de contacto con la realidad facilitando la observación directa de las actividades humanas; favorecen el encuentro de grupos culturales diferentes y distintos componentes de la realidad social, permiten la participación, invitan a la acción y facilitan la relación con otras poblaciones o territorios.

Las salidas del aula constituyen una actividad de colaboración entre la escuela y el entorno social y cultural. La escuela es un agente cultural activo dentro de la comunidad y abre sus puertas a los agentes educativos extraescolares (familia, medios de comunicación, instituciones educativas extraescolares) que le ayudan en la concreción de su propio proyecto educativo.

2 Araceli Vilarrasa Cunillé, "Las salidas escolares. Una estrategia para la integración curricular del estudio del medio local", en Educación primaria. Orientaciones y recursos (6 - 12 años), eds. Joaquín Gairín Sallán e Isabel M. Vizcaíno Timón (Barcelona: Praxis, 2002), 1-20.

3 María del Rosario Caballero, Inicios de la historia del arte en España: la Institución Libre de Enseñanza (1876-1936) (Madrid: CSIC, 2003).

4 Vilarrasa, Las salidas escolares, 1-20. 
De este modo, el entorno, desde la perspectiva escolar, tiene tres funciones: objeto de estudio, laboratorio de experiencias y contexto de la educación ${ }^{5}$.

Continuando con las bondades y beneficios de las salidas del aula, para la propia Vilarrasa ${ }^{6}$ salir del aula supone la posibilidad de llevar la escuela a aquellos espacios en los que se hace posible la vivencia de experiencias culturales y al contexto en el que la información adquiere su sentido. El alumnado, mediante esa experiencia y vivencia directa, puede transformar dicha información en conocimiento mediante un proceso de reelaboración cognitiva, beneficiando la autonomía y personalidad del aprendiz.

Cuando se pretende que el discente observe, busque, manipule y explique lo que ve o descubre, las salidas del aula son el recurso adecuado para ello $^{7}$, convirtiéndose así en un método oportuno para la construcción del conocimiento.

Las salidas escolares también facilitan la convivencia, el conocimiento entre profesorado y alumnado, ayudan a despertar el interés de este, que se convierte en sujeto activo y participativo y refuerza su pertenencia al grupo. Se tornan además como el marco perfecto para trabajar cualidades como la de formar personas socialmente críticas, desarrollar el conocimiento reflexivo y el pensamiento crítico, además de valores y actitudes democráticos ${ }^{8}$.

Para Ávila ${ }^{9}$, tanto las teorías del inductivismo como del constructivismo tienen en las salidas del aula una buena herramienta para el aprendizaje. Desde el inductivismo y su aprendizaje por descubrimiento, las salidas son buenas porque, comprendiendo los métodos, se tiene un conocimiento adecuado de la materia, porque la información que se le da al alumnado no es el resultado del conocimiento experto, porque piensa por sí mismo, estando a veces en desacuerdo, lo que le ayuda en la comprensión del objeto de la visita. Desde el constructivismo y su descubrimiento dirigido, es bueno salir porque se

5 Vilarrasa, Las salidas escolares, 1-20.

6 Araceli Vilarrasa Cunillé, "Salir del Aula. Reapropiarse del contexto", Íber: Didáctica de las Ciencias Sociales, Geografía e Historia, n. ${ }^{\circ} 36$ (2003): 13-26.

7 Pilar Benejam Arguimbau, “Salir del aula”, Íber: Didáctica de las Ciencias Sociales, Geografía e Historia, n. ${ }^{\circ} 36$ (2003): 7-13.

8 Rosa María Medir i Huerta, "Salir de la escuela: entre la tradición y la educación ambiental para la sostenibilidad”, Íber. Didáctica de las Ciencias Sociales, Geografía e Historia, n. 36 (2003): 26-35.

9 Rosa María Ávila Ruiz, "La función de los itinerarios en la enseñanza y el aprendizaje del patrimonio histórico-artístico. Una reflexión didáctica”, Íber. Didáctica de las Ciencias Sociales, Geografía e Historia, n. ${ }^{\circ} 36$ (2003): 36-46. 
reconoce la actitud exploradora y curiosa del aprendizaje del ser humano, se fomenta la autonomía personal, la creatividad y el sentido crítico y se facilita la integración de diversas dimensiones del aprendizaje.

Una buena y correcta organización de una salida en la que se ponga al alumnado en contacto directo con la realidad mediante la observación rigurosa, se contrasten las ideas previas de los discentes, se adquiera sentido crítico, exista correlación entre las actividades de aula y la realidad observada y se consiga la implicación de los aprendices en la transformación del entorno que visitan, requiere de tres pasos fundamentales ${ }^{10}$. Una fase inicial de actividades realizadas en el aula anteriores a la visita, en las que se hagan aflorar los conocimientos previos y se trabaje con nuevas informaciones, estimulando la curiosidad a través de preguntas, proyecciones, lecturas y explicaciones. Una segunda fase de actividades durante el desarrollo de la visita con el fin de aplicar lo aprendido en el aula y analizar los elementos más significativos. Finalmente, una tercera fase de actividades de conclusión en el aula en las que se organice y sistematice lo aprendido antes y después de la visita, se incorpore a sus esquemas cognitivos y se presente al resto de compañeros mediante un trabajo que les ayude en su maduración personal.

Un tipo de salida escolar de gran calado es el itinerario, es decir, un recorrido por diferentes puntos de interés con elementos de valor patrimonial, cultural o natural, entre los que destaca el itinerario urbano, que centra su recorrido en una ciudad o en parte de la misma ${ }^{11}$. A través de él se tiende a buscar el desarrollo de la capacidad de observación del complejo urbano, el desarrollo de la movilidad urbana mediante el empleo de planos y fomentar la reflexión en torno a los fenómenos de índole social, política, económica o cultural que han ocurrido en la ciudad, que se convierte en una fuente de primer orden, ya que se atestiguan en ella el paso del tiempo y los cambios históricos ${ }^{12}$. Para alcanzar con éxito estos objetivos, es necesario planificar un buen itinerario, teniendo en cuenta que hay que evitar la existencia de muchos puntos de parada, el excesivo ruido o tráfico; saber elegir el lugar con la mejor vista del elemento

\footnotetext{
10 Ávila, "La función de los itinerarios", 36-46.

${ }^{11}$ Yolanda Insa, "Itinerarios urbanos, recursos y materiales didácticos para explicar la ciudad”, Íber, Didáctica de las Ciencias Sociales, Geografía e Historia, n. 32 (2002): 89-95.

${ }^{12}$ Ricardo Ruiz Pérez, "El patrimonio histórico. Propuesta didáctica”, en Didáctica de las Ciencias Sociales, Geografía e Historia en la enseñanza secundaria obligatoria, ed. Antonio Luis García Ruiz (Granada: Grupo Editorial Universitario, 1997), 360-401.
} 
en cuestión. Objetivos que deben comprender una didáctica interdisciplinar y poner en juego diferentes sentidos, como la vista, el oído o el olfato ${ }^{13}$.

Desde nuestra óptica, entendemos que las salidas escolares permiten, entre otros, objetivos como el fomento del sentido de la observación del paisaje como fuente de información geográfica e histórica en la que se reflejan todas las actuaciones humanas, la percepción del espacio, el espíritu crítico, la creatividad, el aprecio por los valores del pasado, el respeto del entorno y la dimensión ética que ha de estar en la base de toda actuación relativa al patrimonio.

Por otro lado, si el fin de toda educación y de todo sistema educativo es formar a los jóvenes en los aspectos fundamentales de la vida, como son el respeto a las diferentes culturas, la conciencia de ser ciudadanos y la necesidad de vivir en democracia; las salidas y visitas culturales, a través de una pedagogía del patrimonio, han de considerarse también como un factor importante de tolerancia, convivencia pacífica e integración social. Se hace necesario, desde la enseñanza formal, desarrollar programas pedagógicos en los que se fomente la sensibilización de los jóvenes ante el patrimonio cultural, para resaltar aquellos valores históricos, artísticos y éticos que en él se encuentran representados, enseñando el respeto a las diferentes culturas y el desarrollo de la tolerancia y la eliminación de cualquier desigualdad y exclusión que pueda darse dentro de la sociedad.

Todos los beneficios hasta ahora mencionados son los que han permitido que, en la educación obligatoria, la realización y el surgimiento de propuestas de salidas e itinerarios didácticos suelan ser habituales ${ }^{14}$, tal y como señala Molina $^{15}$, e incluso que existan investigaciones sobre su empleo ${ }^{16}$. Sin embargo, su uso no es para nada común en la formación universitaria (con alguna excepción como lo señala Moreno ${ }^{17}$ ), a pesar de que, como ya subrayara

13 Ávila, “La función de los itinerarios", 36-46.

${ }^{14}$ Pedro Miralles Martínezy Pilar Rivero Gracia, "Propuestas de innovación para la enseñanza de la historia en Educación Infantil”, Reifop 15, n. ${ }^{\circ} 1$ (2012): 81-90.

${ }^{15}$ Sebastián Molina Puche, «El museo “La casa encantada” de Briones en la enseñanza de la historia: propuesta para una salida escolar para educación primaria», Contextos Educativos. Revista de Educación, n. ${ }^{\circ} 13$ (2010): 71-82.

${ }^{16}$ José Monteagudo Fernández y Pedro Miralles Martínez, Utilización didáctica del patrimonio mediante las salidas escolares. Los niveles de bachillerato - COU en la Región de Murcia (Saarbrücken: Publicia, 2014).

${ }^{17}$ Juan Ramón Moreno Vera, “Geovicente: la enseñanza de la Geografía con códigos QR", en XIV Jornades de Xarxes d'Investigació en Docència Universitària. Investigació,innovaciói ensenyament 
Rodríguez ${ }^{18}$, entre los métodos que el profesorado universitario puede utilizar en sus clases para resaltar la dimensión educativa de su profesión está el de itinerarios didácticos. Es aquí, precisamente, cuando nuestro trabajo viene a cubrir una laguna en la experimentación y publicación de salidas del aula con alumnado universitario, en concreto en la didáctica de las ciencias sociales y, dentro de ella, en la didáctica de la historia del arte.

Y ello por tres razones. La primera es que consideramos que la formación en historia del arte en el futuro profesorado de educación primaria es necesaria para lograr personas y profesionales cultos que lleven a las aulas del mañana la enseñanza de los elementos patrimoniales del entorno próximo del alumnado. Pero para que sea así, tal y como indicaba Lozano ${ }^{19}$, hay que proporcionar a los futuros maestros experiencias para reflexionar y apreciar el pasado que se encuentra a su alrededor a fin de que logren hacer sus propios proyectos pedagógicos y ponerlos en práctica.

Esta idea resulta más relevante todavía si tenemos en cuenta que la mayoría de las programaciones anuales de cualquier centro incluyen salidas al entorno y visitas a exposiciones o museos, y que, como han puesto de relieve Monteagudo y Oliveros ${ }^{20}$, a pesar de que los docentes de Ciencias Sociales afirman que realizan salidas fuera del aula para el tratamiento del patrimonio siguiendo el esquema de actividades antes, durante y después de la misma, también es cierto que este mismo profesorado asegura que carece de formación específica sobre la didáctica del patrimonio; lógico, por otra parte, si se tiene en cuenta la escasa importancia de la historia del arte en el currículum español, como se ha visto líneas arriba.

En segundo lugar, con el conocimiento directo de elementos patrimoniales e histórico-artísticos también buscamos que el futuro profesorado programe actividades que lleven al alumnado a aprender más allá del aula, en palabras de

universitari: enfocaments pluridisciplinaris, eds. María Teresa Tortosa Ybáñez, Salvador Grau Company y José Daniel Álvarez Teruel (Alicante: Universitat d'Alacant, Institut de Ciències de l'Educació, 2016), 87-99.

${ }^{18}$ Martín Rodríguez Rojo, "El componente educativo en el rol del profesor universitario", Revista electrónica interuniversitaria de formación del profesorado 2, n. ${ }^{\circ} 1$ (1999): 117-128.

${ }^{19}$ Lozano, "Patrimonio, arte y didáctica”, 7

${ }^{20}$ José Monteagudo Fernández y Carmen Oliveros Ortuño, "La didáctica del patrimonio en las aulas. Un análisis de las prácticas docentes”, RevistaUnes. Universidad, Escuela y Sociedad, n. ${ }^{\circ} 1$ (2016): 64-79. 
Melgar y Silvio ${ }^{21}$, ampliando los límites de acceso al conocimiento a través de propuestas educativas como las citadas salidas del aula e itinerarios didácticos, un tipo de actividades que potencian el aprendizaje significativo a partir de la realidad más próxima del alumnado, integrando experiencia, acción y contexto, desarrollando los procedimientos de observación y análisis, relacionando práctica y teoría y teniendo al alumnado como centro de acción a través del trabajo en grupo.

Además, un profesorado debidamente formado en contenidos históricoartísticos puede pensar en las tareas a realizar durante y después de la visita y no quedare solamente en los aspectos organizativos de la salida ${ }^{22}$.

Finalmente, de esta forma, la escuela hace verdaderamente suyo el papel de institución compensatoria de las desventajas iniciales del alumnado carente de medios o estímulos socio-familiares que le permitan conocer, disfrutar y valorar los bienes patrimoniales, como indican Bourdieu y Darbe ${ }^{23}$.

\section{Metodología}

\section{Participantes en la experiencia didáctica}

En cuanto a la muestra de participantes en la acción didáctica en la que se pretendía enseñar aspectos de la Edad Media a través del aprendizaje situado y el patrimonio de la ciudad de Murcia, se trató de 65 estudiantes del tercer curso del grado de Educación Primaria de la Universidad de Murcia, de los cuales 15 eran varones (el 23.07\%) y 50, mujeres (76.92\% de la muestra). Las edades de los participantes se encuentran entre los 20 y 21 años.

\section{Hipótesis y objetivos de investigación}

$\mathrm{Al}$ iniciar esta investigación se partió de la hipótesis del desconocimiento del alumnado de educación superior del patrimonio que le rodea y la escasa conexión que hace el alumnado entre este y la etapa medieval en la ciudad de Murcia.

${ }^{21}$ María Melgar y Silvio Donolo, "Salir del aula... Aprender de otros contextos: patrimonio natural, museos e Internet”, Revista Eureka sobre enseñanza y divulgación de las ciencias 8, n. 3 (2011): 323-333.

22 Jenaro Guisasola y Maite Morentin, "Concepciones del profesorado sobre visitas escolares a museos de ciencias", Enseñanza de las ciencias 28, n. ${ }^{\circ}$ 1(2010): 127-140.

${ }^{23}$ Pierre Bourdieu y Alain Darbel, El amor al arte. Los museos europeos y su público (Barcelona: Paidós Estética, 2003). 
El principal objetivo que persigue esta investigación es analizar los resultados de una experiencia didáctica basada en las salidas de campo, en la que el alumnado visita el centro histórico de la ciudad de Murcia para conocer su patrimonio medieval, la historia de la ciudad y su pasado histórico-artístico que aún perdura.

El objetivo secundario, aunque para los investigadores muy importante, fue trazar un itinerario coherente, válido para el objetivo principal, y conseguir que nuestro alumnado mejore su aprendizaje sobre el patrimonio de la ciudad y la época medieval.

\section{Instrumentos de análisis}

Metodológicamente la investigación se basó en el análisis cualitativo de las respuestas de los estudiantes a dos cuestionarios: uno, pre-test, con el cual se trató de detectar los conocimientos previos del alumnado antes de afrontar la experiencia didáctica; y otro cuestionario, post-test, para analizar la progresión de aprendizaje del alumnado, en el que se incluían las mismas preguntas que en el primero, para así trazar unos resultados comparativos entre lo que sabía y lo que había aprendido. Cabe destacar que el cuestionario post-test se realizó un mes después de la salida del aula.

Para el análisis comparativo de los resultados del pre-test y el post-test, se agruparon las respuestas del alumnado en distintos ítems, lo que nos permitió una lectura cuantitativa según lo que expresaron los propios estudiantes.

Las preguntas fueron las siguientes:

1. ¿Sabrías decir en cuál época histórica se funda la ciudad de Murcia?

2. ¿Existe todavía una muralla medieval en la ciudad de Murcia?

3. ¿Qué es un alcázar?

4. ¿Sabes dónde se ubicaba el antiguo zoco y mercado medieval de Murcia?

5. ¿Qué es un gremio?

6. En una mezquita, ¿qué es el mihrab?

Las cuestiones de los dos test usados en la investigación tienen que ver con aspectos de la Edad Media que se trabajarían en la experiencia didáctica, desde cuestiones relativas a la Murcia medieval islámica como la fundación de la ciudad o la presencia de los dos alcázares, hasta preguntas sobre la Murcia medieval cristiana como el emplazamiento de la plaza del mercado o las calles gremiales del centro de la ciudad. 


\section{Propuesta didáctica: un itinerario por la Murcia medieval}

El itinerario didáctico que se realizó en esta experiencia didáctica constó de cinco paradas, en las que se incluyeron dos museos: el de Santa Clara (parada inicial del recorrido) y el de San Juan de Dios (última parada del itinerario). Además, se propusieron otras paradas urbanas en la plaza de Santo Domingo, las "Cuatro esquinas" -intersección entre las calles Trapería y Platería-y, por último, la catedral de Murcia.

A continuación, se van a analizar las distintas paradas y los contenidos de la Edad Media que se trabajaron en cada una de ellas; así mismo, se propusieron preguntas para que el alumnado ampliase la información de la visita.

\section{Punto de salida: Alcázar Seguir o menor (convento de Santa Clara)}

El comienzo de la visita medieval a la ciudad de Murcia se situó fuera del recinto de la muralla, en el antiguo alcázar menor de la ciudad, donde pasaban las vacaciones los gobernantes de la misma. Se trataba de un edificio de recreo, ordenado a través de un patio con su alberca central y vegetación. A ambos lados tenía pórticos con arcos donde descansar a la sombra. Tras la conquista cristiana el edificio pasó a manos de la Orden de las Clarisas, lo que ha provocado numerosos cambios en su estructura.

Para aprender más... debes estar atento y seguir las explicaciones de guías y profesores. Indaga, pregunta, lee y encuentra la respuesta para las siguientes preguntas:

- ¿A qué siglos pertenecen los restos hallados en Santa Clara?

- ¿Por qué son importantes y en qué se observa dicha importancia?

- ¿Qué espacios medievales se han recuperado en Santa Clara? Algunos son del palacio en época hudí y otros del palacio en época mardanisí.

\section{Plaza de Santo Domingo}

La plaza de Santo Domingo es hoy uno de centros neurálgicos de la ciudad, pero en época medieval destacó por ser el espacio dedicado al zoco o mercado. Tras la definitiva conquista cristiana de la ciudad por parte del rey Jaime I de Aragón tras la sublevación mudéjar, en 1266 el rey Alfonso X de Castilla otorgó a Murcia el derecho a celebrar los jueves el día de mercado (como actualmente se sigue haciendo), aunque en el siglo XX cambió el espacio dejando de ser Santo Domingo para trasladarse al barrio de La Fama. 
Por hacer bien y merced al Concejo de Murcia, porque sean más ricos y más abonados, les damos y otorgamos para siempre, que hagan mercado en su villa cada semana en día de jueves. [...] todos cuantos a este mercado vinieren o vayan a venir con todas sus mercaderías y con todos sus confines se les respeten sus derechos y que ninguno sea hollado de las prendas ni de los embargos en ninguna manera a ellos, si no es por voluntad propia o por fiaduría ${ }^{24}$.

Para aprender más... debes estar atento y seguir las explicaciones de guías y profesores. Indaga, pregunta, lee y encuentra la respuesta para las siguientes preguntas:

- ¿Dónde aparecía el antiguo zoco o mercado medieval de la ciudad, dentro de sus muros o fuera de ellos?

\section{Las cuatro esquinas}

El cruce de las calles Trapería y Platería en pleno centro histórico de Murcia se conoce como "las cuatro esquinas". Este céntrico punto de la villa medieval era el lugar donde confluían dos de los gremios más importantes de la Murcia cristiana: el de los traperos que comerciaban con telas y el de los plateros que se dedicaban a la joyería y la orfebrería. No son las únicas calles que conservan el nombre de su gremio en la ciudad, ya que también existen las calles de Vidrieros, Jabonerías o Frenería.

Para aprender más... debes estar atento y seguir las explicaciones de guías y profesores. Indaga, pregunta, lee y encuentra la respuesta para las siguientes preguntas:

- La actividad gremial ha quedado reflejada en el trazado urbano de la Murcia actual, ¿además de la trapería y la platería, conoces algún gremio más que todavía mantenga su nombre en las calles de Murcia?

- ¿A qué se dedicaban?

\section{Mezquita Aljama (catedral de Murcia)}

El espacio que actualmente ocupa la catedral de Murcia estuvo previamente ocupado por la mezquita mayor de la ciudad, conservando, por tanto, la función religiosa del espacio. Aunque en la Edad Media cristiana el templo se transformó en un edificio gótico (con fachadas -visible el gótico en la "Puerta

${ }^{24}$ Antonio Botías, «El mercado de los jueves cumple siete siglos», La Verdad, 29 de mayo de 2016. 
de los Apóstoles"- y partes renacentistas y barrocas), todavía conservamos algunos restos de la antigua mezquita en el Museo de la catedral de Murcia.

Para aprender más... debes estar atento y seguir las explicaciones de guías y profesores. Indaga, pregunta, lee y encuentra la respuesta para las siguientes preguntas:

- ¿Existía alguna otra mezquita en la ciudad de Murcia?

\section{Parada final: oratorio del Alcázar Mayor (iglesia de San Juan de Dios)}

La alcazaba era el punto neurálgico del gobierno de la ciudad y donde vivía la persona encargada de la gestión de la ciudad y el reino. Era un recinto fortificado dentro de la propia muralla de la ciudad medieval. Esta función de gobierno se sigue cumpliendo ahora ya que el ayuntamiento se ubica en el mismo espacio; a pesar de eso, la antigua alcazaba era de mayor tamaño y, además, en uno de sus extremos se encontraba el Alcázar Mayor, o el palacio, donde vivía la familia real y su corte. Allí encontramos su oratorio particular donde se conserva el arco del mirhab.

En esta parada podremos observar también uno de los lienzos de la muralla medieval de la ciudad de Murcia. No es el único, ya que el trazado de la muralla está bien delimitado y se conservan numerosos tramos a lo largo de la ciudad (muchos están en garajes, parkings y sótanos de edificios). Podemos observar qué disposición tenía la muralla que rodeaba la ciudad, ya que constaba de una muralla y una antemuralla. Además, estaba jalonada por hasta 95 torres defensivas coronadas por almenas y existían 6 puertas o portillos de entrada a la ciudad, que aumentaron a 12 tras la conquista cristiana.

Para aprender más... debes estar atento y seguir las explicaciones de guías y profesores. Indaga, pregunta, lee y encuentra la respuesta para las siguientes preguntas:

- En el oratorio real de San Juan de Dios, ¿podía rezar cualquier ciudadano? ¿Quién lo hacía allí?

- En la iglesia de San Juan de Dios, encontraremos la imagen de uno de los cristos más antiguos de la región de Murcia. ¿Cuál es? ¿A qué estilo artístico pertenece? 


\section{Resultados}

Por lo que respecta a los resultados de la investigación, se van a analizar de forma comparada las respuestas del alumnado tanto en el pre-test como en el posttest, agrupándolas en ítems que se repetían para una comparación cuantitativa.

En cuanto a la primera de las cuestiones, se les preguntaba si sabían en qué época histórica había sido fundada la ciudad de Murcia. Aunque la gran mayoría de los estudiantes lo tenían claro ya en el pre-test, es una de las cuestiones en la que, gracias a la experiencia didáctica sobre el patrimonio, más se ha notado la progresión de aprendizaje en nuestro alumnado.

Como se puede observar en la Figura 1, los resultados del pre-test evidencian que el 60\% del alumnado conocía el origen medieval de la ciudad de Murcia, fundada en el año 825 por Abderramán II; sin embargo, aún un 40\% se decidía por otro origen distinto de la ciudad. Los importantes restos romanos y tardorromanos en la región (Cartagena, sin ir más lejos, como uno de los focos principales de la romanización de la península) hace que aún existiese un 33\% de los estudiantes que pensaban que Murcia tiene también un origen latino (y, en menor medida, un $5 \%$ pensaba que es renacentista y un $2 \%$ de origen griego).

Figura 1. Resultados del pre-test sobre el origen de la ciudad.

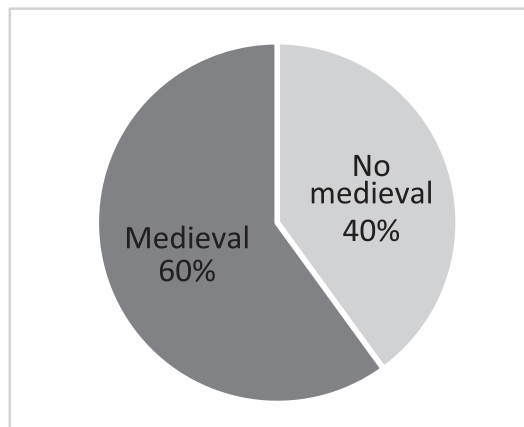

Fuente: Elaboración propia

En la Figura 2 se puede analizar el resultado en el post-test sobre la misma cuestión del origen y fundación de la ciudad, una vez llevada ya a cabo la actividad de la salida de campo y la enseñanza a través del patrimonio. En esta ocasión, el origen medieval de la ciudad representa el 96\% de las respuestas del alumnado, un 36\% más que en el pre-test, mientras que el posible origen romano de la ciudad ahora ya no tiene ninguna presencia, solo un $4 \%$ se decanta por otras opciones como renacentista. 
Figura 2. Resultados del post-test sobre el origen de la ciudad

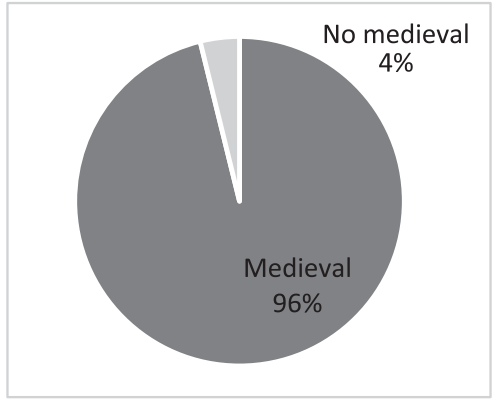

Fuente: Elaboración propia

La mejoría del alumnado en esta cuestión se debe a que la fundación de la ciudad fue uno de los temas tratados in situ durante la visita, ya que la misma se comenzaba en el Alcázar Menor de la ciudad, fundado en época islámica, que fue el periodo en que se creó la ciudad.

Por lo que respecta a la segunda pregunta sobre si existe o no muralla medieval en la ciudad de Murcia, también se observa en los resultados la progresión del aprendizaje, aunque cabe señalar que los resultados previos ya eran muy positivos, al contestar favorablemente a su existencia un $75 \%$ del alumnado, mientras que una vez hecha la salida didáctica (y visitado uno de los tramos de muralla que se encuentra bajo la iglesia de San Juan de Dios), ese porcentaje que indica su existencia asciende al 92\% de los estudiantes.

Otra de las preguntas en la que es posible analizar la progresión de aprendizaje del alumnado, gracias a la experiencia didáctica, es la tercera pregunta que les cuestionaba si sabían lo que era un alcázar.

En la Figura 3 se muestran los resultados del pre-test y, aunque de nuevo, una mayoría, el 52\% del alumnado, sabía lo que es -constituye una visita turística muy habitual en ciudades como Granada, Almería, Córdoba o Sevilla y además es una palabra relativamente común en nuestra lengua, con numerosos topónimos esparcidos por nuestra geografía- existen todavía dudas y confusión sobre su posible uso como fortaleza - el 40\% de las respuestas-, ya que algunos de los alcázares mayores que habían visitado previamente se asocian a una fortificación mayor, la alcazaba, edificaciones todavía presentes en muchas ciudades españolas y que, en ocasiones, suponen el uso de fortaleza y de palacio al mismo tiempo. 
Figura 3. Resultados pre-test sobre el concepto de alcázar

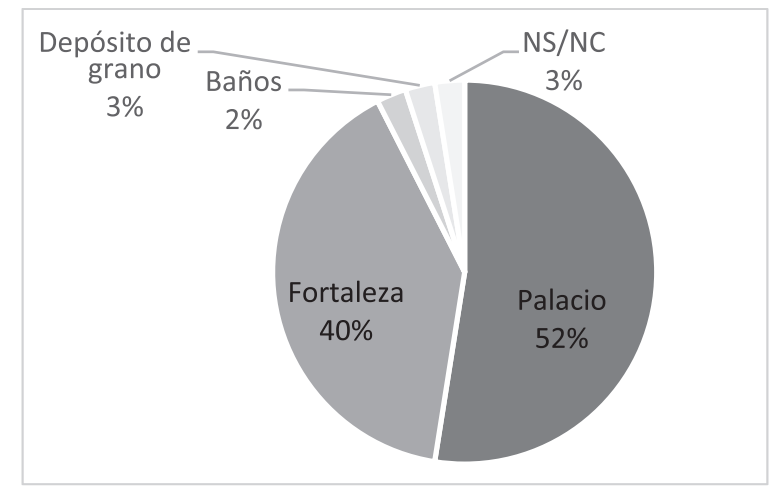

Fuente: Elaboración propia

Sin embargo, los resultados del post-test sobre la misma cuestión subrayan cómo la experiencia didáctica ha ayudado a aclarar este concepto ligado a la arquitectura medieval islámica. En la Figura 4 se puede observar cómo un $74 \%$ de los estudiantes asociaban alcázar al concepto de palacio o residencia (al haber visitado en la experiencia el alcázar menor de la ciudad de Murcia), lo que suponía un $22 \%$ más que en el pre-test; mientras que las dudas sobre su uso como fortaleza descendían al 15\% de las respuestas.

Figura 4. Resultados post-test sobre el concepto alcázar

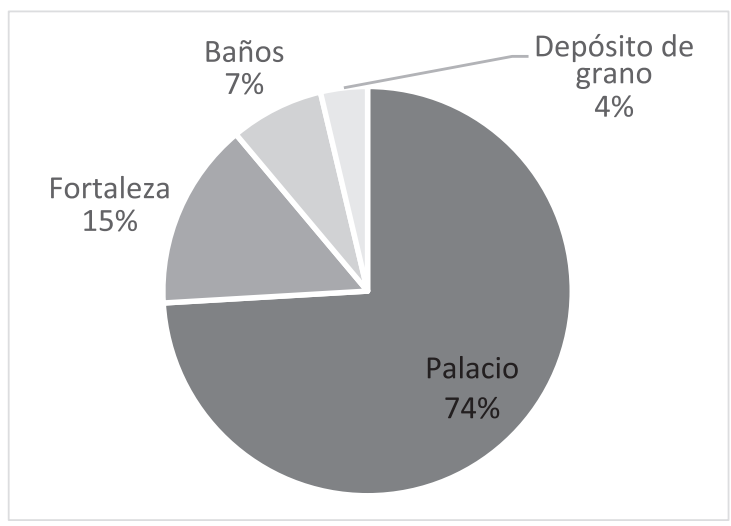

Fuente: Elaboración propia 
A continuación, se van a analizar los resultados que corresponden a la cuarta pregunta, en la que nos adentramos en el corazón medieval de la Murcia cristiana: la plaza del mercado y de la inquisición, la actual plaza de Santo Domingo.

En la Figura 5, se pueden observar los resultados del pre-test en el que se les pedía que situasen la medieval plaza del mercado en la ciudad. La mayoría de estudiantes la situaban en el actual espacio de alguna plaza de abastos, un $32 \%$, mientras que su correcto emplazamiento en la actual Santo Domingo fue apenas contestado por un 25\% del alumnado. La presencia de respuestas relativas a otros emplazamientos como la plaza de la Catedral -20\%- o La Fama-13\%-, donde actualmente se ubica el mercado, son todavía notables.

Figura 5. Resultados pre-test sobre el mercado medieval

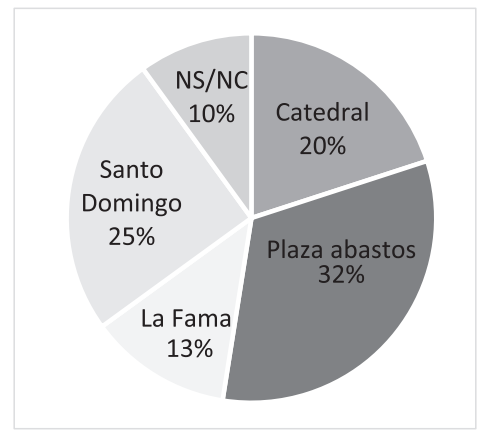

Fuente: Elaboración propia

La Figura 6, por el contrario, muestra los resultados del post-test y evidencia de nuevo el aprendizaje del alumnado a través del estudio del patrimonio y la salida de campo. En esta ocasión, la plaza de Santo Domingo, centro neurálgico del comercio medieval, apareció en el $84 \%$ de las respuestas del alumnado frente al 25\% del pre-test. El resto de respuestas casi desaparecen, descendiendo el porcentaje del alumnado que pensaba que se encuentra en la actual plaza de abastos al 8\%, frente al 32\% del pre-test. Aunque al día de hoy, dicha plaza sigue siendo el corazón comercial de la ciudad, no tiene un uso como mercado público en la actualidad, por lo que este aspecto se trabajó durante la visita para que el alumnado hiciera un trabajo de abstracción apoyado por fotografías antiguas en las que aparece el mercado popular instalado en esta misma plaza. 
Figura 6. Resultados post-test sobre el mercado medieval

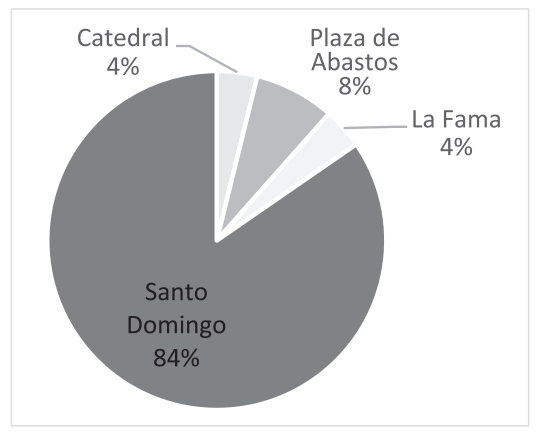

Fuente: Elaboración propia

En relación a la pregunta número 5 de nuestra experiencia sobre qué es un gremio, los resultados previos y posteriores son muy similares, ya que antes de comenzar la experiencia y visitar las principales calles de Murcia que conservan los nombres gremiales, el alumnado ya conocía qué significaba esta palabra y qué clase de asociación representaban los gremios. Los resultados positivos sobre estas uniones de comerciantes que pervivieron en España hasta el siglo XIX se encuentran cercanos al 100\%, tanto antes como después del itinerario didáctico.

Por último, se analizan los resultados sobre la sexta y última pregunta, en la que se les cuestionaba sobre qué es un mihrab, el nicho en el muro de la quibla que marca el lugar donde descansan las escrituras islámicas durante la oración y hacia donde los musulmanes dirigen sus rezos en la mezquita.

Figura 7. Resultados pre-test sobre el mihrab

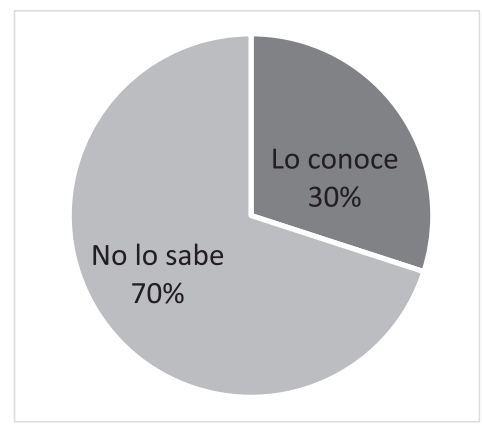

Fuente: Elaboración propia 
En la Figura 7 se puede observar como en el pre-test apenas el 30\% de los estudiantes conocen este concepto; esto es debido a que es una palabra que no ha pervivido en nuestra lengua y que responde a un rito religioso que el alumnado desconoce.

Sin embargo, los resultados se revierten cuando se analizan las contestaciones del post-test, una vez ya hecha la experiencia didáctica en la que se visitó el arco del mihrab del oratorio del alcázar mayor de la ciudad de Murcia que se conserva en la iglesia de San Juan de Dios.

Figura 8. Resultados post-test sobre mihrab

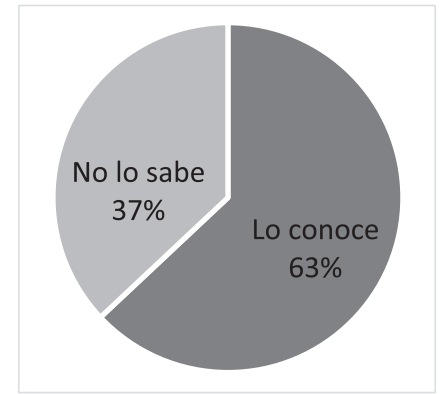

Fuente: Elaboración propia

La Figura 8 muestra cómo, en esta ocasión, hasta el 63\% del alumnado era capaz de contestar correctamente qué es un mihrab, mientras que las respuestas erróneas descendieron de un 70\%, en el pre-test, hasta un 37\% en el test posterior a la visita. En este sentido, y ante un concepto dificultoso como el del mihrab, realizar la visita al oratorio de San Juan de Dios y ver el arco del mihrab fue fundamental para explicar esta mejoría en el aprendizaje del alumnado.

Así pues, los resultados de algunas de las principales preguntas realizadas antes de comenzar la experiencia didáctica muestran cómo, aunque el alumnado conocía en su mayoría los aspectos principales de la Murcia medieval, todavía existían dudas y confusiones en algunos puntos, hechos que se han solventado, en gran medida, gracias al estudio in situ del patrimonio a través del itinerario didáctico por la Murcia medieval. 


\section{Conclusiones}

Cuando se iniciaba esta experiencia didáctica para aprender la historia del arte de la época medieval en la ciudad de Murcia a través de un itinerario didáctico y su patrimonio, se cuestionaba si el alumnado sería capaz de mejorar su aprendizaje gracias a las salidas de campo y al análisis directo del patrimonio.

Los test realizados para analizar la progresión de aprendizaje de los estudiantes han mostrado unos resultados muy satisfactorios, habiendo conseguido los discentes mejorar su aprendizaje gracias al itinerario didáctico y a la visita del patrimonio.

Algunos de los resultados son bastante elocuentes en lo que respecta a su aprendizaje ya que, por ejemplo, las contestaciones sobre el origen medieval de la ciudad mejoraron de un 60 a 96\%, el concepto de alcázar pasó de un 52 a un $74 \%$, la ubicación del antiguo mercado de la ciudad pasó a ser reconocida de un 25 a un $84 \%$ y el concepto de mihrab, que era bastante desconocido antes de hacer la salida, mejoró de un 30 a un 63\%.

Esta mejoría en el aprendizaje de los discentes se fundamenta en el uso de la salida de campo como método de enseñanza y en el poder de atracción que ejerce el patrimonio como documento fehaciente en nuestros días de contenidos conceptuales de la Edad Media. El alumnado se siente cómodo y motivado al realizar una actividad de enseñanza-aprendizaje fuera del aula y, además, en el caso de la Didáctica de las Ciencias Sociales, son especialmente interesantes ya que la conexión de los contenidos históricos que se ven en clase con la realidad que les rodea en su día a día - el patrimonio de su ciudad-es un hecho que permite crear nuevas conexiones y relaciones que mejoran el aprendizaje de nuestros estudiantes.

Así pues, el objetivo principal de nuestra investigación, que era analizar los resultados de esta experiencia didáctica, ha quedado completado de manera satisfactoria al conseguir, mediante la salida de campo, una clara mejoría en el aprendizaje de nuestro alumnado.

\section{Referencias bibliográficas}

\section{Fuentes secundarias}

Ávila Ruiz, Rosa María. "La función de los itinerarios en la enseñanza y el aprendizaje del patrimonio histórico-artístico. Una reflexión didáctica”, Íber. Didáctica de las Ciencias Sociales, Geografía e Historia, n. 36 (2003): 36-46.

Benejam Arguimbau, Pilar. “Salir del aula”, Íber: Didáctica de las Ciencias Sociales, Geografía e Historia, n. ${ }^{\circ} 36$ (2003): 7-13. 
Bourdieu, Pierre y Alain Darbel. El amor al arte. Los museos europeos y su público. Barcelona: Paidós Estética, 2003.

Caballero, María del Rosario. Inicios de la historia del arte en España: la Institución Libre de Enseñanza (1876-1936). Madrid: CSIC, 2003.

Guisasola, Jenaro y Maite Morentin. "Concepciones del profesorado sobre visitas escolares a museos de ciencias”, Enseñanza de las ciencias 28, n. ${ }^{\circ}$ 1(2010): 127-140.

Insa, Yolanda. "Itinerarios urbanos, recursos y materiales didácticos para explicar la ciudad”, Íber, Didáctica de las Ciencias Sociales, Geografía e Historia, n. ${ }^{\circ} 32$ (2002): 89-95.

Lozano López, Esther. "Patrimonio, arte y didáctica de las Ciencias Sociales. Análisis y reflexiones sobre una estrategia de aprendizaje en el marco de innovación docente", Clío. History and History Teaching, n. 39 (2010).

Melgar, María y Silvio Donolo. "Salir del aula... Aprender de otros contextos: patrimonio natural, museos e Internet”, Revista Eureka sobre enseñanza y divulgación de las ciencias $8, \mathrm{n}^{\circ} 3$ (2011): 323-333.

Medir i Huerta, Rosa María. "Salir de la escuela: entre la tradición y la educación ambiental para la sostenibilidad”, Íber. Didáctica de las Ciencias Sociales, Geografía e Historia, n. ${ }^{\circ} 36$ (2003): 26-35.

Miralles Martínez, Pedro y Pilar Rivero Gracia. "Propuestas de innovación para la enseñanza de la historia en Educación Infantil”, Reifop 15, n. ${ }^{\circ} 1$ (2012): 81-90.

Monteagudo Fernández, José y Carmen Oliveros Ortuño. "La didáctica del patrimonio en las aulas. Un análisis de las prácticas docentes”, RevistaUnes. Universidad, Escuelay Sociedad, n. 1 (2016): 64-79.

Monteagudo Fernández, José y Pedro Miralles Martínez. Utilización didáctica del patrimonio mediante las salidas escolares. Los niveles de bachillerato-COU en la región de Murcia. Saarbrücken: Publicia, 2014.

Molina Puche, Sebastián. «El museo "La casa encantada” de Briones en la enseñanza de la historia: propuesta para una salida escolar para educación primaria», Contextos Educativos. Revista de Educación, n. ${ }^{\circ} 13$ (2010): 71-82.

Moreno Vera, Juan Ramón. "Geovicente: la enseñanza de la Geografía con códigos QR”. En XIV Jornades de Xarxes d'Investigació en Docència Universitària. Investigació, innovació iensenyament universitari: enfocaments pluridisciplinaris, eds. María Teresa Tortosa Ybáñez, Salvador Grau Company y José Daniel Álvarez Teruel. Alicante: Universitat d'Alacant, Institut de Ciències de l'Educació, 2016.

Rodríguez Rojo, Martín. "El componente educativo en el rol del profesor universitario", Revista electrónica interuniversitaria de formación del profesorado 2, n. $^{\circ} 1$ (1999): 117-128.

Ruiz Pérez, Ricardo. "El patrimonio histórico. Propuesta didáctica”. En Didáctica de las Ciencias Sociales, Geografía e Historia en la enseñanza secundaria obligatoria, ed. Antonio Luis García Ruiz. Granada: Grupo Editorial Universitario, 1997.

Vilarrasa Cunillé, Araceli. "Salir del Aula. Reapropiarse del contexto”, Íber: Didáctica de las Ciencias Sociales, Geografía e Historia, n. 36 (2003): 13-26. 
Aprender historia del arte mediante salidas didácticas. Una experiencia en educación superior Juan Ramón Moreno-Vera - José Monteagudo Fernández

Vilarrasa Cunillé, Araceli. “Las salidas escolares. Una estrategia para la integración curricular del estudio del medio local”. En Educación primaria. Orientaciones y recursos (6-12 años), eds. Joaquín Gairín Sallán e Isabel M.. Vizcaíno Timón. Barcelona: Praxis, 2002 